\title{
Homotopy Analysis Method for Equations
} of the Type $\nabla^{2} u=b(x, y)$ and $\nabla^{2} u=b(x, y, u)$

\author{
Selcuk Yildirim \\ Department of Electrical and Electronics Engineering, Siirt University, Siirt, Turkey \\ Email: syildirim@siirt.edu.tr
}

Received January 2015

\begin{abstract}
In this paper, the homoto pyanalysis method (HAM) is presented to solve some of engineering problems. The homotopy analysis method is applied in obtaining exact solutions for equations of the type $\nabla^{2} u=b(x, y)$ and $\nabla^{2} u=b(x, y, u)$ on an elliptical domain. Exact solutions are presented for several examples involving to demon strate the applic ability and efficiency of HAM.
\end{abstract}

Keywords

Homotopy Analysis Method, Engineering Problems, Exact Solutions

\section{Introduction}

The homotopy analysis method is developed in 1992 by Liao [1]-[8]. It is an analytical approach to get the series solution of linear and nonline arpartial differential equations. The difference with the other perturbation methods is that this method is independent of small/large physical parameters. It also provides a simple way to ensure the convergence of series solution [9]. This method has been successfully applied to solve many linear and non linear partial differential equationsin various fields of science and engineering by many authors [1]-[16]. The homotopy analysis method is useful and efficient for obtaining both analytical and numerical approximations of linear or nonlinear differential equations. In this study, we will concentrate on exact solutions for equations type of $\nabla^{2} u=b(x, y)$ and $\nabla^{2} u=b(x, y, u)$ frequently used in applied and engineering mathematics.

\section{The Engineering Equations on an Elliptical Domain}

We refer to the problem given by Partridge and Brebbia [17]. Consider the following engineering equations

$$
\frac{\partial^{2} u}{\partial x^{2}}+\frac{\partial^{2} u}{\partial y^{2}}=b(x, y)
$$

How to cite this paper: Yildirim, S. (2015) Homotopy Analysis Method for Equations of the Type $\nabla^{2} u=b(\mathrm{x}, \mathrm{y})$ and $\nabla^{2} u=b(\mathrm{x}, \mathrm{y}, \mathrm{u})$. Journal of Applied Mathematics and Physics, 3, 391-398. http://dx.doi.org/10.4236/iamp.2015.34049 


$$
\frac{\partial^{2} u}{\partial x^{2}}+\frac{\partial^{2} u}{\partial y^{2}}=b(x, y, u)
$$

where $b(x, y)$, that is, considered to be a known function of position and $b(x, y, u)$ will be considered as a known function of the potential.

In all applications, the domain bounded by the ellipse given in Figure 1 will be used. The boundary condition is the Dirichlet condition with $u=0$ on the boundary.

The equation of the ellipse is

$$
\frac{x^{2}}{4}+y^{2}=1
$$

\section{Homotopy Analysis Method}

We apply the HAM to equations of the type $\nabla^{2} u=b(x, y)$ and $\nabla^{2} u=b(x, y, u)$ with Dirichlet boundary condition. We consider the following differential equation

$$
N[u(x, y)]=0
$$

where $N$ is a linear operator, $x$ and $y$ are independent variables, $u(x, y)$ is an unknown function. In HAM, the zeroth-orderde formation equation is constructed as

$$
(1-p) \mathcal{L}\left[\phi(x, y ; p)-u_{0}(x, y)\right]=p \hbar N[\phi(x, y ; p)]
$$

where

$$
\phi(x, y ; p)=u_{0}(x, y)+\sum_{m=1}^{\infty} u_{m}(x, y) p^{m}
$$

$\mathcal{L}$ is an auxiliary parameter, $u_{0}(x, y)$ is an initial guess, $\hbar \neq 0$ is an auxiliary parameter and $p \in[0,1]$ is the embedding parameter. Applying the homotopy-derivative [4]

$$
u_{m}(x, y)=\left.\frac{1}{m !} \frac{\partial^{m} \phi(x, y ; p)}{\partial p^{m}}\right|_{p=0}
$$

To both sides of Equation (5), we get the following $m$ th-order deformation equation

$$
\mathcal{L}\left[u_{m}(x, y)-\chi_{m} u_{m-1}(x, y)\right]=\hbar \Re_{m-1}
$$

where

$$
\mathfrak{R}_{m-1}=\left.\frac{1}{(m-1) !} \frac{\partial^{m-1} N[\phi(x, y ; p)]}{\partial p^{m-1}}\right|_{p=0}
$$

And

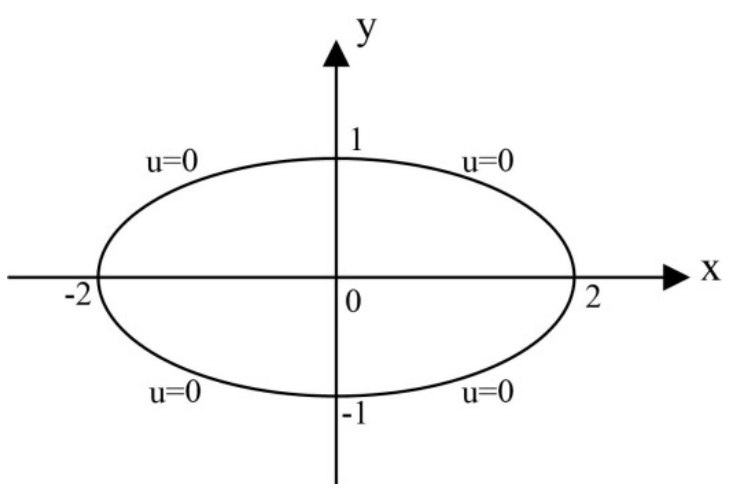

Figure 1. Elliptical domain with Dirichlet boundary condition. 


$$
\chi_{m}= \begin{cases}0, & m \leq 1, \\ 1, & m>1 .\end{cases}
$$

Note that $u_{m}(x, y)$ for $m \geq 1$ can be obtained by solving the linear Equation (8) with linear boundary conditions that come from original problem. If the power series Equation (6) of $\phi(x, y ; p)$ converges at $p=1$, then we gets the following series solution:

$$
u(x, y)=u_{0}(x, y)+\sum_{m=1}^{\infty} u_{m}(x, y)
$$

\section{Applications}

We apply Homotopy Analysis Method to equations of the type $\nabla^{2} u=b(x, y)$ and $\nabla^{2} u=b(x, y, u)$, as follows:

Equation (1) suggests that we define an equation of linear operator as

$$
N[\phi(x, y ; p)]=\frac{\partial^{2} \phi(x, y ; p)}{\partial x^{2}}+\frac{\partial^{2} \phi(x, y ; p)}{\partial y^{2}}+b(x, y)
$$

And Equation (2) suggests that we define an equation of linear operator as

$$
N[\phi(x, y ; p)]=\frac{\partial^{2} \phi(x, y ; p)}{\partial x^{2}}+\frac{\partial^{2} \phi(x, y ; p)}{\partial y^{2}}+b(x, y, \phi(x, y ; p))
$$

Using the above definitions, the zeroth-orderde formation equation is constructed as

$$
(1-p) \mathcal{L}\left[\phi(x, y ; p)-u_{0}(x, y)\right]=p \hbar N[\phi(x, y ; p)]
$$

Applying the homotopy-derivative to the zeroth-orderde formation equation, we obtain the following $m$ thorderde formation equations

$$
\mathcal{L}\left[u_{m}(x, y)-\chi_{m} u_{m-1}(x, y)\right]=\hbar \Re_{m-1}
$$

Since $m \geq 1, \chi_{m}=1$, now the solution of the $m$ th-orderde formation equation becomes

$$
u_{m}(x, y)=u_{m-1}(x, y)+\hbar \mathcal{L}^{-1}\left[\Re_{m-1}\right]
$$

where

$$
\begin{gathered}
\mathfrak{R}_{m-1}=\frac{\partial^{2} u_{m-1}(x, y)}{\partial x^{2}}+\frac{\partial^{2} u_{m-1}(x, y)}{\partial y^{2}}+b(x, y) \\
\mathfrak{R}_{m-1}=\frac{\partial^{2} u_{m-1}(x, y)}{\partial x^{2}}+\frac{\partial^{2} u_{m-1}(x, y)}{\partial y^{2}}+b\left(x, y, u_{m-1}(x, y)\right)
\end{gathered}
$$

Example 1. Consider the equation of the type $\nabla^{2} u=b$

$$
\frac{\partial^{2} u}{\partial x^{2}}+\frac{\partial^{2} u}{\partial y^{2}}=-2
$$

With initial guess

$$
u_{0}(x, y)=-\frac{4}{5} y^{2}+\frac{4}{5}
$$

using HAM, were cursively obtain

$$
\begin{gathered}
u_{1}(x, y)=\hbar \int_{0}^{x} \int_{0}^{x} \frac{\partial^{2} u_{0}(x, y)}{\partial x^{2}} \mathrm{~d} x \mathrm{~d} x+\hbar \int_{0}^{x} \int_{0}^{x} \frac{\partial^{2} u_{0}(x, y)}{\partial y^{2}} \mathrm{~d} x \mathrm{~d} x+\hbar \int_{0}^{x} \int_{0}^{x} 2 \mathrm{~d} x \mathrm{~d} x \\
u_{1}(x, y)=\hbar \frac{1}{5} x^{2}
\end{gathered}
$$




$$
\begin{gathered}
u_{2}(x, y)=u_{1}(x, y)+\hbar \int_{0}^{x} \int_{0}^{x} \frac{\partial^{2} u_{1}(x, y)}{\partial x^{2}} \mathrm{~d} x \mathrm{~d} x+\hbar \int_{0}^{x} \int_{0}^{x} \frac{\partial^{2} u_{1}(x, y)}{\partial y^{2}} \mathrm{~d} x \mathrm{~d} x \\
u_{2}(x, y)=\left(\hbar+\hbar^{2}\right) \frac{1}{5} x^{2} \\
u_{3}(x, y)=u_{2}(x, y)+\hbar \int_{0}^{x} \int_{0}^{x} \frac{\partial^{2} u_{2}(x, y)}{\partial x^{2}} \mathrm{~d} x \mathrm{~d} x+\hbar \int_{0}^{x} \int_{0}^{x} \frac{\partial^{2} u_{2}(x, y)}{\partial y^{2}} \mathrm{~d} x \mathrm{~d} x \\
u_{3}(x, y)=\left(\hbar+2 \hbar^{2}+\hbar^{3}\right) \frac{1}{5} x^{2} \\
u(x, y)=-\frac{4}{5} y^{2}+\frac{4}{5}+\hbar \frac{1}{5} x^{2}+\left(\hbar+\hbar^{2}\right) \frac{1}{5} x^{2}+\left(\hbar+2 \hbar^{2}+\hbar^{3}\right) \frac{1}{5} x^{2}+\cdots
\end{gathered}
$$

When $\hbar=-1$, we obtain the exact solution as follows:

$$
\begin{aligned}
& u(x, y)=-\frac{1}{5} x^{2}-\frac{4}{5} y^{2}+\frac{4}{5} \\
& u(x, y)=-\frac{4}{5}\left(\frac{x^{2}}{4}+y^{2}-1\right)
\end{aligned}
$$

Example 2. Consider the equation of the type $\nabla^{2} u=b(x, y)$

$$
\frac{\partial^{2} u}{\partial x^{2}}+\frac{\partial^{2} u}{\partial y^{2}}=-x
$$

with initial guess

$$
u_{0}(x, y)=-\frac{x^{3}}{14}+\frac{2 x}{7}
$$

using HAM, were cursively obtain

$$
\begin{gathered}
u_{1}(x, y)=\hbar \int_{0}^{y} \int_{0}^{y} \frac{\partial^{2} u_{0}(x, y)}{\partial x^{2}} \mathrm{~d} y \mathrm{~d} y+\hbar \int_{0}^{y} \int_{0}^{y} \frac{\partial^{2} u_{0}(x, y)}{\partial y^{2}} \mathrm{~d} y \mathrm{~d} y+\hbar \int_{0}^{y} \int_{0}^{y} x \mathrm{~d} y \mathrm{~d} y \\
u_{1}(x, y)=\hbar \frac{2 x}{7} y^{2} \\
u_{2}(x, y)=u_{1}(x, y)+\hbar \int_{0}^{y} \int_{0}^{y} \frac{\partial^{2} u_{1}(x, y)}{\partial x^{2}} \mathrm{~d} y \mathrm{~d} y+\hbar \int_{0}^{y} \int_{0}^{y} \frac{\partial^{2} u_{1}(x, y)}{\partial y^{2}} \mathrm{~d} y \mathrm{~d} y \\
u_{3}(x, y)=\left(\hbar+\hbar^{2}\right) \frac{2 x}{7} y^{2} \\
u_{3}(x, y)=\left(\hbar+2 \hbar^{2}+\hbar^{3}\right) \frac{2 x}{7} y^{2} \\
\vdots x, y)+\hbar \int_{0}^{y} \int_{0}^{y} \frac{\partial^{2} u_{2}(x, y)}{\partial x^{2}} \mathrm{~d} y \mathrm{~d} y+\hbar \int_{0}^{y} \int_{0}^{y} \frac{\partial^{2} u_{2}(x, y)}{\partial y^{2}} \mathrm{~d} y \mathrm{~d} y \\
u(x, y)=-\frac{x^{3}}{14}+\frac{2 x}{7}+\hbar \frac{2 x}{7} y^{2}+\left(\hbar+\hbar^{2}\right) \frac{2 x}{7} y^{2}+\left(\hbar+2 \hbar^{2}+\hbar^{3}\right) \frac{2 x}{7} y^{2}+\cdots
\end{gathered}
$$


When $\hbar=-1$, we obtain the exact solution as follows:

$$
\begin{aligned}
& u(x, y)=-\frac{x^{3}}{14}+\frac{2 x}{7}-\frac{2 x}{7} y^{2} \\
& u(x, y)=-\frac{2 x}{7}\left(\frac{x^{2}}{4}+y^{2}-1\right)
\end{aligned}
$$

Example 3. Consider the equation of the type $\nabla^{2} u=b(x, y, u)$

$$
\frac{\partial^{2} u}{\partial x^{2}}+\frac{\partial^{2} u}{\partial y^{2}}=-u
$$

with initial guess

$$
u_{0}(x, y)=x
$$

using HAM, were cursively obtain

$$
\begin{gathered}
u_{1}(x, y)=\hbar \int_{0}^{x} \int_{0}^{x} \frac{\partial^{2} u_{0}(x, y)}{\partial x^{2}} \mathrm{~d} x \mathrm{~d} x+\hbar \int_{0}^{x} \int_{0}^{x} \frac{\partial^{2} u_{0}(x, y)}{\partial y^{2}} \mathrm{~d} x \mathrm{~d} x+\hbar \int_{0}^{x} \int_{0}^{x} u_{0}(x, y) \mathrm{d} x \mathrm{~d} x \\
u_{1}(x, y)=\hbar \frac{x^{3}}{3 !} \\
u_{2}(x, y)=u_{1}(x, y)+\hbar \int_{0}^{x} \int_{0}^{x} \frac{\partial^{2} u_{1}(x, y)}{\partial x^{2}} \mathrm{~d} x \mathrm{~d} x+\hbar \int_{0}^{x} \int_{0}^{x} \frac{\partial^{2} u_{1}(x, y)}{\partial y^{2}} \mathrm{~d} x \mathrm{~d} x+\hbar \int_{0}^{x} \int_{0}^{x} u_{1}(x, y) \mathrm{d} x \mathrm{~d} x \\
u_{2}(x, y)=\left(\hbar+\hbar^{2}\right) \frac{x^{3}}{3 !}+\hbar^{2} \frac{x^{5}}{5 !} \\
u_{3}(x, y)=\left(\hbar+3 \hbar^{2}+2 \hbar^{3}\right) \frac{x^{3}}{3 !}+\left(\hbar^{2}+\hbar^{3}\right) \frac{x^{5}}{5 !}+\hbar^{3} \frac{x^{7}}{7 !} \\
\vdots \\
u(x, y)=x+\hbar \frac{x^{3}}{3 !}+\left(\hbar+\hbar^{2}\right) \frac{x^{3}}{3 !}+\hbar^{2} \frac{x^{5}}{5 !}+\left(\hbar+3 \hbar^{2}+2 \hbar^{3}\right) \frac{x^{3}}{3 !}+\left(\hbar^{2}+\hbar^{3}\right) \frac{x^{5}}{5 !}+\hbar^{3} \frac{x^{7}}{7 !}+\cdots
\end{gathered}
$$

For $\hbar=-1$, we obtained the closed form series solution as

$$
\begin{gathered}
u(x, y)=x-\frac{x^{3}}{3 !}+\frac{x^{5}}{5 !}-\frac{x^{7}}{7 !}+\cdots \\
u(x, y)=\sin x
\end{gathered}
$$

which is the exact solution.

Example 4. Consider the equation of the type $\nabla^{2} u=b(x, y, u)$

$$
\frac{\partial^{2} u}{\partial x^{2}}+\frac{\partial^{2} u}{\partial y^{2}}=-\frac{\partial u}{\partial x}
$$

with initial guess

$$
u_{0}(x, y)=1-x
$$

using HAM, were cursively obtain 


$$
\begin{gathered}
u_{1}(x, y)=\hbar \int_{0}^{x} \int_{0}^{x} \frac{\partial^{2} u_{0}(x, y)}{\partial x^{2}} \mathrm{~d} x \mathrm{~d} x+\hbar \int_{0}^{x} \int_{0}^{x} \frac{\partial^{2} u_{0}(x, y)}{\partial y^{2}} \mathrm{~d} x \mathrm{~d} x+\hbar \int_{0}^{x} \int_{0}^{x} \frac{\partial u_{0}(x, y)}{\partial x} \mathrm{~d} x \mathrm{~d} x \\
u_{1}(x, y)=-\hbar \frac{x^{2}}{2 !}(x, y)=u_{1}(x, y)+\hbar \int_{0}^{x} \int_{0}^{x} \frac{\partial^{2} u_{1}(x, y)}{\partial x^{2}} \mathrm{~d} x \mathrm{~d} x+\hbar \int_{0}^{x} \int_{0}^{x} \frac{\partial^{2} u_{1}(x, y)}{\partial y^{2}} \mathrm{~d} x \mathrm{~d} x+\hbar \int_{0}^{x} \int_{0}^{x} \frac{\partial u_{1}(x, y)}{\partial x} \mathrm{~d} x \mathrm{~d} x \\
u_{3}(x, y)=u_{2}(x, y)+\hbar \int_{0}^{x} \int_{0}^{x} \frac{\partial^{2} u_{2}(x, y)}{\partial x^{2}} \mathrm{~d} x \mathrm{~d} x+\hbar \int_{0}^{x} \int_{0}^{x} \frac{\partial^{2} u_{2}(x, y)}{\partial y^{2}} \mathrm{~d} x \mathrm{~d} x+\hbar \int_{0}^{x} \int_{0}^{x} \frac{\partial u_{2}(x, y)}{\partial x} \mathrm{~d} x \mathrm{~d} x \\
u_{3}(x, y)=-\left(\hbar+2 \hbar^{2}+\hbar^{3}\right) \frac{x^{2}}{2 !}-2\left(\hbar^{2}+\hbar^{3}\right) \frac{x^{3}}{3 !}-\hbar^{3} \frac{x^{4}}{4 !} \\
\vdots \\
u(x, y)=1-x-\hbar \frac{x^{2}}{2 !}-\left(\hbar+\hbar^{2} \frac{x^{3}}{3 !} \frac{x^{2}}{2 !}-\hbar^{2} \frac{x^{3}}{3 !}-\left(\hbar+2 \hbar^{2}+\hbar^{3}\right) \frac{x^{2}}{2 !}-2\left(\hbar^{2}+\hbar^{3}\right) \frac{x^{3}}{3 !}-\hbar^{3} \frac{x^{4}}{4 !}-\cdots\right.
\end{gathered}
$$

For $\hbar=-1$, we obtained the closed form series solution as

$$
\begin{gathered}
u(x, y)=1-x+\frac{x^{2}}{2 !}-\frac{x^{3}}{3 !}+\frac{x^{4}}{4 !}-\cdots \\
u(x, y)=\mathrm{e}^{-x}
\end{gathered}
$$

which is the exact solution.

Example 5. Consider the equation of the type $\nabla^{2} u=b(x, y, u)$

$$
\frac{\partial^{2} u}{\partial x^{2}}+\frac{\partial^{2} u}{\partial y^{2}}=-\frac{\partial u}{\partial x}-\frac{\partial u}{\partial y}
$$

with initial guess

$$
u_{0}(x, y)=\mathrm{e}^{-x}+1-y
$$

using HAM, were cursively obtain

$$
\begin{gathered}
u_{1}(x, y)=\hbar \int_{0}^{y} \int_{0}^{y} \frac{\partial^{2} u_{0}(x, y)}{\partial x^{2}} \mathrm{~d} y \mathrm{~d} y+\hbar \int_{0}^{y} \int_{0}^{y} \frac{\partial^{2} u_{0}(x, y)}{\partial y^{2}} \mathrm{~d} y \mathrm{~d} y+\hbar \int_{0}^{y} \int_{0}^{y} \frac{\partial u_{0}(x, y)}{\partial x} \mathrm{~d} y \mathrm{~d} y+\hbar \int_{0}^{y} \int_{0}^{y} \frac{\partial u_{0}(x, y)}{\partial y} \mathrm{~d} y \mathrm{~d} y \\
\begin{array}{c}
u_{2}(x, y)=-\hbar \frac{y^{2}}{2 !} \\
+u_{1}(x, y)+\hbar \int_{0}^{y} \int_{0}^{y} \frac{\partial^{2} u_{1}(x, y)}{\partial x^{2}} \mathrm{~d} y \mathrm{~d} y+\hbar \int_{0}^{y} \int_{0}^{y} \frac{\partial^{2} u_{1}(x, y)}{\partial y^{2}} \mathrm{~d} y \mathrm{~d} y+\hbar \int_{0}^{y} \int_{0}^{y} \frac{\partial u_{1}(x, y)}{\partial x} \mathrm{~d} y \mathrm{~d} y \\
+\hbar \int_{0}^{y} \int_{0}^{y} \frac{\partial u_{1}(x, y)}{\partial y} \mathrm{~d} y \mathrm{~d} y, \\
u_{2}(x, y)=-\left(\hbar+\hbar^{2}\right) \frac{y^{2}}{2 !}-\hbar^{2} \frac{y^{3}}{3 !}
\end{array}
\end{gathered}
$$




$$
\begin{aligned}
& u_{3}(x, y)= u_{2}(x, y)+\hbar \int_{0}^{y} \int_{0}^{y} \frac{\partial^{2} u_{2}(x, y)}{\partial x^{2}} \mathrm{~d} y \mathrm{~d} y+\hbar \int_{0}^{y} \int_{0}^{y} \frac{\partial^{2} u_{2}(x, y)}{\partial y^{2}} \mathrm{~d} y \mathrm{~d} y+\hbar \int_{0}^{y} \int_{0}^{y} \frac{\partial u_{2}(x, y)}{\partial x} \mathrm{~d} y \mathrm{~d} y \\
&+ \hbar \int_{0}^{y} \int_{0}^{y} \frac{\partial u_{2}(x, y)}{\partial y} \mathrm{~d} y \mathrm{~d} y, \\
& u_{3}(x, y)=-\left(\hbar+2 \hbar^{2}+\hbar^{3}\right) \frac{y^{2}}{2 !}-2\left(\hbar^{2}+\hbar^{3}\right) \frac{y^{3}}{3 !}-\hbar^{3} \frac{y^{4}}{4 !} \\
& \vdots \\
& u(x, y)=\mathrm{e}^{-x}+1-y-\hbar \frac{y^{2}}{2 !}-\left(\hbar+\hbar^{2}\right) \frac{y^{2}}{2 !}-\hbar^{2} \frac{y^{3}}{3 !}-\left(\hbar+2 \hbar^{2}+\hbar^{3}\right) \frac{y^{2}}{2 !}-2\left(\hbar^{2}+\hbar^{3}\right) \frac{y^{3}}{3 !}-\hbar^{3} \frac{y^{4}}{4 !}-\cdots
\end{aligned}
$$

For $\hbar=-1$, we obtained the closed form series solution as

$$
\begin{gathered}
u(x, y)=\mathrm{e}^{-x}+1-y+\frac{y^{2}}{2 !}-\frac{y^{3}}{3 !}+\frac{y^{4}}{4 !}-\cdots \\
u(x, y)=\mathrm{e}^{-x}+\mathrm{e}^{-y}
\end{gathered}
$$

which is the exact solution.

\section{Conclusion}

In this paper, the homotopy analysis method has been applied to solve some of engineering problems defined on an elliptical domain. Exact solutions for equations of the type $\nabla^{2} u=b(x, y)$ and $\nabla^{2} u=b(x, y, u)$ are obtained using the HAM. Obviously for $\hbar=-1$ the obtained solutions are as the same Reference [17]. There sults show that HAM is very efficient technique in finding the exact solutions for equations of the type $\nabla^{2} u=b(x, y)$ and $\nabla^{2} u=b(x, y, u)$ having wide applications in engineering mathematics.

\section{References}

[1] Liao, S.J. (2005) Comparison between the Homotopy Analysis Method and Homotopy Perturbation Method. Applied Mathematics and Computation, 169, 1186-1194.

[2] Liao, S.J. (2004) On the Homotopy Analysis Method for Nonlinear Problems. Applied Mathematics and Computation, 147, 499-513.

[3] Liao, S.J. (2010) An Optimal Homotopy-Analysis Approach for Strongly Nonlinear Differential Equations. Communications in Nonlinear Science and Numerical Simulation, 15, 2003-2016.

[4] Liao, S.J. (2009) Notes on the Homotopy Analysis Method: Some Definitions and Theorems. Communications in Nonlinear Science and Numerical Simulation, 14, 983-997. http://dx.doi.org/10.1016/j.cnsns.2008.04.013

[5] Liao, S.J. (2005) An Analytic Approach to Solve Multiple Solutions of a Strongly Nonlinear Problem. Applied Mathematics and Computation, 169, 854-865.

[6] Liao, S.J. (2003) Beyond Perturbation: Introduction to the Homotopy Analysis Method. Chapman and Hall/CRC Press, Boca Raton.

[7] Liao, S.J. (2012) Homotopy Analysis Method in Nonlinear Differential Equations. Springer, New York.

[8] Liao, S.J. (1992) Proposed Homotopy Analysis Techniques for the Solution of Nonlinear Problems. Ph.D. Thesis, Shanghai Jiao Tong University, Shanghai.

[9] Das, S., Vishal, K., Gupt, P.K. and Ray, S.S. (2011) Homotopy Analysis Method for Solving Fractional Diffusion Equation. International Journal of Applied Mathematics and Mechanics, 7, 28-37.

[10] Song, L. and Zhang, H. (2007) Application of Homotopy Analysis Method to Fractional Kdv-Burgers-Kuramoto Equation. Physics Letters A, 367, 88-94.

[11] Abdulaziz, O., Hashim, I. and Saif, A. Solutions of Time-Fractional PDEs by Homotopy Analysis Method. Differential Equations and Nonlinear Mechanics, 2008, Article ID: 686512.

[12] Ganjiani, M. (2010) Solution of Nonlinear Fractional Differential Equations Using Homotopy Analysis Method. Applied Mathematical Modelling, 34, 1634-1641. http://dx.doi.org/10.1016/j.apm.2009.09.011

[13] Abidi, F. and Omrani, K. (2010) The Homotopy Analysis Method for Solving the Fornberg-Whitham Equation and 
Comparison with Adomian's Decomposition Method. Computers \& Mathematics with Applications, 59, 2743-2750.

[14] Molabahrami, A. and Khani, F. (2009) The Homotopy Analysis Method to Solve the Burgers-Huxley Equation. Nonlinear Analysis: Real World Applications, 10, 589-600. http://dx.doi.org/10.1016/j.nonrwa.2007.10.014

[15] Ghanbari, B. (2104) An Analytical Study for (2+1)-Dimensional Schrödinger Equation. The Scientific World Journal, 2014, Article ID: 438345.

[16] Inc, M. (2007) On Exact Solution of Laplace Equation with Dirichlet and Neumann Boundary Conditions by Homotopy Analysis Method. Physics Letters A, 365, 412-415.

[17] Patridge, P.W., Brebbia, C.A. and Wrobel, L.C. (1992) The Dual Reciprocity Boundary Element Method. CMP, Elsevier Applied Scince, New York. 